\title{
Método de corrección de factores: alternativa para la valoración de suelos. Caso Arequipa Metropolitana
}

\author{
Factor correction method: alternative for soil valuation. \\ Arequipa Metropolitan Case
}

\section{Método de correção de fator: alternativa para avaliação de solos. Caso Metropolitano de Arequipa}

\author{
- Silvia Noelia López Ortega \\ slopezo@unsa.edu.pe \\ Código ORCID: 0000-0002-8402-8451
}

- Edgar García Anco

egarciaan@unsa.edu.pe

Código ORCID: 0000-0002-7534-2086

Universidad Nacional de Agustín de Arequipa, Perú

Artículo recibido 1 de diciembre 2020 / Arbitrado y aceptado 14 de diciembre 2020 / publicado 18 de diciembre 2020

\section{RESUMEN}

Debido al crecimiento económico y poblacional, la adquisición de viviendas o terrenos para la construcción en Arequipa Metropolitana ha requerido, la aplicación de regulaciones para evitar el fenómeno de la especulación y la segregación geográfica, al respecto el presente artículo tiene por propósito aplicar el método de corrección de factores para la valoración de suelos urbanos de Aznar y Guijarro. Mediante la comparación de métodos de tasación aplicados por entidades bancarias para créditos hipotecarios y el valor del mercado establecido por el propietario, fueron establecidos siete criterios para la ciudad mencionada con el apoyo de un análisis longitudinal: número y orientación de fachadas, valor por metro cuadrado, recuperación de la inversión, distancia y ubicación a medios de transportes y centros económicos y de servicios básicos, además de establecer que la corrección de factores se encuentra en un 0.92 del valor del mercado.

Palabras clave: Método de corrección de factores; suelos urbanos, valoración

\begin{abstract}
Due to the economic and population growth, the acquisition of houses or land for construction in Metropolitan Arequipa has required the application of regulations to avoid the phenomenon of speculation and geographic segregation, in this regard, this article aims to apply the method of Factor correction for the valuation of urban land in Aznar and Guijarro. By comparing the appraisal methods applied by banks for mortgage loans and the market value established by the owner, seven criteria were established for the city mentioned with the support of a longitudinal analysis: number and orientation of facades, value per square meter, recovery of investment, distance and location to means of transport and economic centers and basic services, in addition to establishing that the factor correction is 0.92 of the market value.
\end{abstract}

Key words: Factor correction metho; urban soils, valuation 


\section{RESUMO}

Devido ao crescimento econômico e populacional, a aquisição de moradias ou terrenos para construção na área metropolitana de Arequipa tem exigido a aplicação de normas para evitar o fenômeno da especulação e segregação geográfica, neste sentido, este artigo visa aplicar o método de correção do fator para a avaliação de terrenos urbanos em Aznar e Guijarro. Comparando os métodos de avaliação aplicados pelos bancos para o crédito à habitação e o valor de mercado estabelecido pelo proprietário, foram estabelecidos sete critérios para o município referido com o apoio de uma análise longitudinal: número e orientação das fachadas, valor por metro quadrado, recuperação do investimento, distância e localização a meios de transporte e centros econômicos e serviços básicos, além de estabelecer que a correção do fator é de 0,92 do valor de mercado.

Palavras-chave: Método de correção de fatores; solos urbanos, valoração

\section{INTRODUCCIÓN}

El crecimiento poblacional y la conformación de grupos familiares demanda en las zonas rurales y/o urbanas la construcción de viviendas, desde cualquier latitud la necesidad por construcciones dignas, con la mayoría de los servicios exigidos por los objetivos de desarrollo sostenible en Agenda 2030 (1). Precisamente dentro del entorno económico y social, el crecimiento por grupos familiares son atribuibles a: la independencia económica, formación familiar, ofertas de empleo, cualidades de las zonas, instituciones educativas. (2). Por esto las condiciones apuntan al desplazamiento a zonas urbanas, conocidas como migraciones internas, llegando a establecerse más del $70 \%$ de la población peruana en áreas urbanas (3).

Haciendo del desarrollo urbanístico una oportunidad, tanto para el vendedor como para el comprador; es claro que la falta de planificación urbanísticas genera disfuncionalidad en la construcción de las viviendas, llegando a prescindir de servicios públicos necesarios para el mantenimiento de una vida digna; por tanto, la ubicación del inmueble, los servicios a disfrutar, el acceso al transporte, a zonas verdes, a seguridad, son factores fundamentales para la adquisición de viviendas; abriendo un panorama para la especulación en los valores bases de la compra de una propiedad (4). La descomposición del valor público con el incremento del bien mismo, indican criterios de interés para parte del grupo de vendedores y la imposibilidad de establecer controles oportunos en el valor de los suelos urbanos (5).

Los estudios de habitabilidad en zona urbanas presentan cualidades recurrentes entorno a la homogeneidad, sostenibilidad y el mono centrismo $(6,7,8)$. Siendo la combinación de las subjetividades del grupo comprador vendedor el resultado del valor del suelo, precisamente en las ciudades con potencialidades de crecimiento económico el valor de zonas residenciales cobra una relación proporcional, por la demanda comercial e industrial en el incremento de sus bienes; siendo aún más privilegiado y valorado el suelo urbano (5). Como afirmación los factores asociados a la plusvalía urbana en la rentabilidad, los servicios públicos (agua), educación (7), inversiones del Estado en obras públicas e inversiones por el sector privado (9), seguridad, precio (10).

La diferencia de precios arraigada por la densificación, la rentabilidad de venta, aumento de los precios por servicios, el bajo aprecio por zonas verdes, ha movilizados las zonas residenciales a terrenos agrícolas adyacentes a las ciudades o centros poblados, y a su vez ocasiona a habitantes urbanos a migrar a zonas donde los precios sean más asequibles a su grupo familiar; todos estos eventos origina un aumento súbito y hasta no creíble del valor de 
los suelo; la situación descrita es evidencia palpable en la zona metropolitana de Arequipa Perú (5), incentivado con tal realidad los entes gubernamentales desarrollan e implementan un plan para el desarrollo y planificación urbana con aplicación desde el 2016 hasta el 2024.

A raíz de las circunstancias el fenómeno de la especulación en la plusvalía en las zonas urbanas residenciales, el mismo, influye negativamente en las oportunidades de los grupos o personas en la adquisición de la propiedad: segregación poblacional, densidad poblacional, planificación urbana deplorable, desmejora en la calidad de vida. (5). El establecimiento de condiciones, normas $\mathrm{y}$ metodologías para una valuación regulada es imperioso; con ello tal sistema de control permitirá frenar tales eventos especulativos, una actualización de metodologías, y establecer medidas para la tasación inmobiliaria

Dentro de este marco, el presente artículo se abordará la utilización de las metodologías y procedimientos utilizados en Arequipa Metropolitana, se contrastarán con el método de corrección de factores de (11), para presentar una metodología de valuación acorde

\section{Corrección por factores (11)}

Proceso en el cual se establecen comparables entre dos o más bienes, que por sus tipologías son similares pero no iguales.

\section{Comparación (14)}

Se compara el activo inmobiliario con otros inmuebles características

semejantes de las cuales se conocen su precio, usando variables explicativas.

a las regulaciones de plusvalías urbanas en Arequipa Metropolitana.

\section{Fundamentación teórica}

El Valor del suelo del suelo es la apreciación de la utilidad de uso y cambio de un terreno cuyas cualidades topográficas permiten el planeamiento habitacional o urbanístico, considerando su tamaño y proporcionalidad (12). Por tanto, el precio monetario por la tierra con fines residenciales, es entendida como la "capitalización de la renta" (5) en complemento con el tiempo esta será incrementada por el valor de uso. Para determinar los condicionantes para la capitalización del suelo: “a) es un mercado de imperfección en la competencia, b) es un mercado de especulación, c) es un mercado complementario del mercado de la construcción y, producto de las anteriores, d) es un mercado fuertemente orientado al alza" (13).

Para establecer la valuación del suelo se aplican dependiendo de la utilidad y tipología de métodos diferentes para comparar los valores del mercado, con el valor del suelo y las regulaciones pertinentes del país en cuestión, a continuación, se presenta un resumen del método de valuación de suelos.

\section{Valores Tipológicos \\ (13)}

Se realiza una valoración económica y de cualidades de un terreno o área con fines urbanísticos desde su geografía, accesibilidad, estructura del suelo y forma del territorio.

\section{Residual}

(15)

Define el valor del
inmueble por la
composición de
diferentes costes, del
suelo, de ra
construcción, los gastos
y beneficios de
promoción.


De acuerdo (11) la valoración de un inmueble urbano consta de variables y subvariables que mejoran el análisis y se articulan al concepto de conglomerado en Proceso Analítico Jerárquico (AHP) entre estas:

1. Entorno urbanístico: categoría de la zona, contaminación, servicios, calidad de establecimientos comerciales, características de la calle, zonas verdes.

2. Características del edificio: fachada, ascensor, portal, portería, conservación de instalaciones.

3. Características de la vivienda: calidad de acabados y materiales, baños, diseño, superficie, altura, iluminación, ventilación, asilamiento, área con construcción y sin, terrazas.

4. Distancia a centros de interés: centro urbano, metro, parada de transporte. (pp.147-148)

Así también se incluye un término importante en los suelos con fines residenciales, la plusvalía urbana, la misma toma dos vertientes: determina la rentabilidad de las zonas para los propietarios $\mathrm{y}$ por sus elevados valores impide el acceso a ciertos sector por parte de familias o personas (5), al igual que el último aspecto en la tasación de los suelos o propiedad urbanas con fines habitacionales se genera un fenómeno negativo en la interrelación del mercado con la plusvalía urbana, el cual es la especulación del terreno, hecho que se beneficia de la oportunidad del valor por uso del suelo y de las fluctuaciones del mercado, con ganancias excedente a las normales en cortos periodos de tiempo, es decir una mayor recuperación de la inversión en corto tiempo (5).

\section{MATERIALES Y MÉTODOS}

Con un enfoque cuantitativo de diseño no experimental longitudinal de tipo descriptivo de alcance exploratorio, fueron determinadas las características residenciales mediante un cuestionario a posibles vendedorescompradores de la zona de Arequipa Metropolitana para el 2017, así mismo se realizó un análisis de documentos para los valores evolutivos de las propiedades desde el 2006 hasta el 2016. La muestra estuvo conformada por los sujetos: compradores y vendedores registrados en la Superintendencia Nacional de los Registro Públicos (SUNARP) quedando distribuidos así cada año: 2006-2007 (372), 2008-2011 (374), 2012 (375), 2013-2015 (374), у 2016 (373). (5)

Los datos fueron organizados en tablas de frecuencia, de acuerdo a las dimensiones de la tabla de operacionalización, y al estudio del periodo 2006 a 2016 permitió establecer los factores comparables; dónde posteriormente fueron seleccionados dos inmuebles con los criterios similares en factores de modificación del valor del suelo: 1) ubicación, 2) orientación, 3) alcance a infraestructura de servicio, 4) fachada, 5) posibilidad de transporte, y 6) tiempo de recuperación de la inversión. Para la aplicación del método de Corrección de factores de (11) se tomó en cuenta: características del predio a valorar, del predio comparable, y el coeficiente corrector al precio.

\section{RESULTADOS}

En el siguiente apartado se muestran los resultados arrojados en el estudio relacionados con las dimensiones desarrolladas en el estudio. (Tabla 1). 
Tabla 1. Operacionalización de las variables

\begin{tabular}{|c|c|c|c|c|}
\hline Variable & Indicadores & Criterio & & Unidad \\
\hline \multirow{8}{*}{ Valuación } & Diferencia entre valor de & $0-100$ & Muy bajo & \multirow{8}{*}{ Soles } \\
\hline & tasación $\quad \mathrm{y}$ valor del & $100-200$ & Bajo & \\
\hline & vendedor & $200-300$ & Medio & \\
\hline & & $300-400$ & Alto & \\
\hline & \multirow{4}{*}{$\begin{array}{l}\text { Diferencia de valuación en } \\
\text { el tiempo }\end{array}$} & Más 400 & Muy alto & \\
\hline & & $0-50$ & Bajo & \\
\hline & & $51-100$ & Medio & \\
\hline & & Más de 100 & Alto & \\
\hline \multirow{19}{*}{$\begin{array}{l}\text { Especulación } \\
\text { del suelo }\end{array}$} & & $0-100 \%$ & Bajo & \multirow{4}{*}{ Porcentaje } \\
\hline & \multirow[t]{3}{*}{ Rentabilidad } & $101 \%-200 \%$ & Medio & \\
\hline & & Más de $200 \%$ & Alto & \\
\hline & & $1 \%-2 \%$ & Bajo & \\
\hline & Comisión de ventas & $2 \%-3 \%$ & Normal & \multirow[t]{3}{*}{ Soles } \\
\hline & \multirow{7}{*}{$\begin{array}{l}\text { Ubicación del centro de } \\
\text { actividades más cercano }\end{array}$} & Más 3\% & Alto & \\
\hline & & $0-0.50 \mathrm{~km}$ & 1.2 & \\
\hline & & $0.51-1.00 \mathrm{~km}$ & 1.1 & \multirow{5}{*}{ Factor } \\
\hline & & $1.00-1.50 \mathrm{~km}$ & 1.0 & \\
\hline & & $1.51-2.00 \mathrm{~km}$ & 0.95 & \\
\hline & & Más de 2.00 km & 0.90 & \\
\hline & & Al este & 1.15 & \\
\hline & \multirow{4}{*}{ Orientación de la fachada } & Al oeste & 1.10 & \multirow{4}{*}{ Soles } \\
\hline & & Al norte & 0.95 & \\
\hline & & Al sur & 0.90 & \\
\hline & & $0-0.50 \mathrm{~km}$ & 1.2 & \\
\hline & \multirow{5}{*}{$\begin{array}{l}\text { Alcance a infraestructura a } \\
\text { servicios básicos }\end{array}$} & $0.51-1.00 \mathrm{~km}$ & 1.1 & \multirow{3}{*}{ Factor } \\
\hline & & $1.00-1.50 \mathrm{~km}$ & 1.0 & \\
\hline & & $1.51-2.00 \mathrm{~km}$ & 0.95 & \\
\hline \multirow[t]{14}{*}{ Valor del suelo } & & Más de $2.00 \mathrm{~km}$ & 0.90 & \\
\hline & & 1 fachada & 1.00 & \multirow{3}{*}{ Factor } \\
\hline & \multirow[t]{2}{*}{ Número de fachadas } & 2 fachadas & 1.10 & \\
\hline & & 3 fachadas & 1.15 & \\
\hline & \multirow{3}{*}{ Segmentación Estatusfera } & Otorgada & 1.05 & \multirow{10}{*}{ Soles } \\
\hline & & No otorgada & 1.00 & \\
\hline & & $0-0.50 \mathrm{~km}$ & 1.2 & \\
\hline & \multirow{4}{*}{ Posibilidad de transporte } & $0.51-1.00 \mathrm{~km}$ & 1.1 & \\
\hline & & $1.00-1.50 \mathrm{~km}$ & 1.0 & \\
\hline & & $1.51-2.00 \mathrm{~km}$ & 0.95 & \\
\hline & & Más de $2.00 \mathrm{~km}$ & 0.90 & \\
\hline & \multirow{3}{*}{$\begin{array}{l}\text { Tiempo de recuperación de } \\
\text { la inversión }\end{array}$} & $0-1$ año & 1.1 & \\
\hline & & $1-5$ años & 1.0 & \\
\hline & & Más de 5 años & 0.9 & \\
\hline
\end{tabular}

Fuente: (5) 
Búsqueda del predio comparable, fue empleada la plataforma "Laencontre" portal con experiencia inmobiliaria de más de dos décadas de funcionamiento. La comparación de factores, el coeficiente es el resultado del producto de los coeficientes aplicables. La aplicación de los coeficientes se determinan el Valor del predio a valorar (VPV), como el resultado del producto de: Coeficiente Corrector Total (CCT), Metro cuadrado comparable (PC/m2) en dólar americano (USD) y área del predio a valorar (aPV). Fórmula: VPV=(CCT)(PC/m^2) (aPV). (Tabla 2).

Tabla 2. Coeficiente corrector aplicable

\begin{tabular}{|c|c|c|c|}
\hline Factores & $\begin{array}{c}\text { Predio a } \\
\text { valorar }\end{array}$ & $\begin{array}{c}\text { Predio } \\
\text { comparable }\end{array}$ & $\begin{array}{c}\text { Coeficiente } \\
\text { corrector aplicable }\end{array}$ \\
\hline Ubicación & $1.42 \mathrm{Km}$ & $0.28 \mathrm{Km}$ & 1.00 \\
\hline Orientación & Norte & Sur & 0.95 \\
\hline Alcance a infraestructura de servicio & $0.72 \mathrm{Km}$ & $1.17 \mathrm{Km}$ & 1.10 \\
\hline Fachadas & 1 & 2 & 1.00 \\
\hline Posibilidad de transporte & $1.09 \mathrm{Km}$ & $0.29 \mathrm{Km}$ & 1.00 \\
\hline Tiempo de recuperación de inversión & 5años & 2años & 0.90 \\
\hline Precio por $\mathrm{m}^{2}$ & & USD 881.66 & $\cdots$ \\
\hline Coeficiente corrector total (CCT) & & & 0.94 \\
\hline
\end{tabular}

Aplicación del VPV: VPV= (CCT) $\left(\mathrm{PC} / \mathrm{m}^{\wedge} 2\right)(\mathrm{aPV})$, el área del predio a valorar es de $80 \mathrm{~m} 2$

$$
\begin{aligned}
& \mathrm{VPV}=(0.94)(881.66)(80.00) \\
& \mathrm{VPV}=66,300.83 \mathrm{USD}
\end{aligned}
$$

Para determinar las diferencias entre los métodos de valuación, la metodología tradicional hace referencia a la tasación la inmueble para la obtención de un crédito hipotecario en una institución bancaria (considerando solo los valores para el terreno), el precio otorgado por el propietario (vendedor) y el método propuesto por la corrección de factores comparables. (Tabla 3).

Tabla 3: Comparación entre los métodos de valuación

\begin{tabular}{ccccc}
\hline & Valor del Predio & $\begin{array}{c}\text { Metodología } \\
\text { tradicional }\end{array}$ & $\begin{array}{c}\text { Otorgado por el } \\
\text { propietario }\end{array}$ & $\begin{array}{c}\text { Metodología } \\
\text { propuesta }\end{array}$ \\
\cline { 2 - 5 } & Valor Total & USD $60,000.00$ & USD $72,00.00$ & USD $66,300.83$ \\
$\begin{array}{c}\text { Especulación } \\
\text { del suelo }\end{array}$ & Área & & $80.00 \mathrm{~m}^{2}$ & \\
& Valor por $\mathrm{m}^{2}$ USD & USD 750.00 & USD 900.00 & USD 828.76 \\
& Valor por $\mathrm{m}^{2} \mathrm{~S} / \mathrm{TC}: 3.30$ & $\mathrm{~S} / 2475.00$ & $\mathrm{~S} / .2970 .00$ & $\mathrm{~S} / .2734 .90$ \\
\hline
\end{tabular}


Considerando dentro del precio otorgado por propietario para la venta en el mercado, que la mitología de corrección obtiene un valor menor o igual al 0.92 al valor mencionado por el propietario; lo cual contribuye a la mitigación de la especulación por la plusvalía urbana.

\section{Discusión}

El establecimiento de patrones uniformes para la valuación de los suelos con fines residenciales, se ven afectados por factores externos a la especulación del mercado, al uso de metodologías tradicionales, y la falta de control gubernamental, hecho que se ve reflejado en zonas o sectores donde el crecimiento económico van a mayor velocidad que la planificación urbanística; existes organizaciones a nivel latinoamericano e Iberoamericano que han determinado normas técnicas para la valuación de suelos, programas $\mathrm{y}$ proyectos de inversión (industriales $\mathrm{y}$ habitabilidad), desglosando metodologías como el método residual dinámico simplificado (15) haciendo tipificaciones en valores de repercusión y valores unitarios; pero detalla la aplicabilidad en situaciones dónde de la forma y situación es más importante que el potencial edificable, es decir con escasa urbanización, condiciones en la cual contradice los factores de influencia tanto de la tasación tradicional por parte de las entidades bancarias, como de los valores subjetivos del mercado por parte del propietario, en el caso de Arequipa Metropolitana.

En España existe una amplia fundamentación legal y regulatoria sobre la valoración de los suelos, sea con finalidades expropiatorias, y de garantía hipotecaria, desde el 2003 se han modificado normativas con reflejo de tres aspectos: metodología unificada, realidad del mercado, y legalidad en la regulación (16), siendo el instrumento estabilizador de los precios de viviendas y terrenos en momentos de inestabilidad, demanda, y la transparencia en adjudicación tanto en situación rural como urbana (17). Al igual en Ecuador la aplicación de normas técnicas para la valoración de los suelos ha permitido establecer precios equilibrados (18) a la realidad del mercado y a las expectativas del propietario, mediante el uso de plataformas como google maps y aplicaciones móviles (19), otros métodos con aplicaciones catastrales con base en programas topográficos en articulación con variables: uso, años de construcción, zonificación, acabado, estructura y valores (estimado e investigado) (13).

En Chile (13) la utilización de métodos cualitativos para suelos urbanos ha establecido controles en los límites de los precios equilibrando el valor del terreno con el valor de la propiedad, busca la preservación del terreno desde la sustentabilidad, con inversiones a mediano y largo plazo; de la misma forma, las necesidades de la transparencias en las plusvalías para el acceso de las familias a una vivienda, nace del agravio del mercado y las herramientas remuneratorias para tasar adecuadamente el hábitat residencial (20) haciendo necesario actualizar y aplicar métodos regulatorios con aplicabilidad inmobiliaria.

\section{CONCLUSIONES}

Las regulaciones en los precios de los terrenos urbanos con fines habitacionales, adquiere mayor relevancia cuando las metodologías presentan falencia o luces para el fenómeno de especulación, el uso de metodologías, adecuadas a características subjetivas o cualitativas para el comprador, brindan una herramienta eficaz para encontrar la congruencia entre el valor del mercado y el valor del suelo. De igual forma, la aplicación de metodologías con apoyo topográfico, y factores tales como: acceso a 
bienes públicos, seguridad, instituciones de salud, educativas, mercados, servicios públicos, fachadas, estructura, entre otros, importantes para el futuro comprador; son esenciales tanto para garantizar el acceso a una vivienda, así como, asegurar las normativas en la planificación urbana y el reglamento de tasaciones del país.

El mencionado método de corrección de factores, radica de una serie de criterios que fueron categorizados, de acuerdo a las necesidades de los habitantes y futuros habitantes de la Arequipa Metropolitana; mediante un estudio coyuntural ejecutado por López (5), estas tipologías permiten estandarizar criterios únicos para cada zona, sea urbana o rural. Y su comparación con los diferentes métodos utilizados, hasta el momento en la zona de interés, permite aseverar un criterio de 0.92 del precio de venta estipulado por el propietario y más consonó con la realidad del mercado.

Por tanto, para la Arequipa Metropolitana se detallan siete factores influyentes en la valuación del suelo urbano: 1) el valor por metro cuadrado en USD y Soles, 2) la ubicación del bien a un centro de actividades, 3) la orientación de la fachada del terreno, 4) la ubicación del bien a una institución u organización: educativa, salud y de compra de víveres y alimentos, 5) la distancia a vías o medios de transporte, 6) capacidad de recuperar la inversión y 7) número de fachadas. $(5,11)$

\section{REFERENCIAS BIBLIOGRÁFICAS}

1. PODS. Perú por los ODS. Perú Sostenible. (2009). http://www.pods.pe/

2. Organización Internacional para Migraciones. Migraciones internas en Perú. https://peru.iom.int/sites/default/files/D ocumentos/Migraciones_Internas.pdf
3. INEI. Perù: Perfil Sociodemográfico. (2017).

https://www.inei.gob.pe/media/MenuRe cursivo/publicaciones_digitales/Est/Lib1 539/cap01.pdf

4. Valdivieso, J. Especulación en el mercado inmobiliario | Inmobiliario | Actualidad | ESAN. Conexión Esan. (2013). https://www.esan.edu.pe/conexion/actu alidad/2013/10/30/especulacionmercado-inmobiliario/

5. López, S. Precio del suelo y metodologías de valaución, una exploración para la regulación de plusvalías urbanas en el caso de Arequipa metropolitana [Universidad Nacional de San Agustín de Arequipa].

(2017). http://repositorio.unsa.edu.pe/bitstream /handle/UNSA/11045/UPloorsn.pdf?sequ ence $=1$ \&isAllowed $=\mathrm{y}$

6. Libertun, N., \& Osorio, René. El efecto del gasto público en el déficit de vivienda en el Perú a nivel municipal (IDB-WP-01112; División de Vivienda y Desarrollo Urbano). $\quad$ (2020). https://publications.iadb.org/publication s/spanish/document/El-efecto-del-gastopublico-en-el-deficit-de-vivienda-en-elPeru-a-nivel-municipal.pdf

7. Quintana, J., Ojeda, A., \& García, J. Factores que explican el valor del suelo urbano. Contexto, XII (16), (2018); 93-108. https://www.researchgate.net/publicatio n/329583996_Factores_que_explican_el_v alor_del_suelo_urbano

8. Vega, V., \& Ruíz, R. Desarrollo sostenible y vivienda digna como punto de progreso social. Sustainable Development and Decent Housing as a Point of Social Progress (2016). 17(1). http://www.scielo.org.co/pdf/agor/v17n 1/v17n1a14.pdf

9. Soria, G. La plusvalía urbana como mecanismo para mejorar el financiamiento de infraestructura pública en la ciudad de Nuevo Chimbote, 2018 [Universidad de san Pedro]. (2018) http://repositorio.usanpedro.edu.pe/bitst ream/handle/USANPEDRO/11007/Tesis_ 60612.pdf?sequence $=1$ \&isAllowed $=y$ 
10. Timaná, J., \& Castañeda, P. Factores determinantes en la selección de vivienda social en el Perú: el caso de Chincha | Publicaciones | ESAN (No. 80; Gerencia Para El Desarrollo). (2019). https://www.esan.edu.pe/publicaciones /serie-gerencia-para-eldesarrollo/2019/factoresdeterminantes-en-la-seleccion-devivienda-social-en-el-peru-el-caso-dechincha/

11. Aznar, J., \& Guijarro, F. Nuevos métodos de valoración: Modelo Multicriterio (Universita). (2012). https://institutodevaluacion.com/wpcontent/uploads/2018/06/NUEVOSMÉTODOS-DE-VALORACIÓN-MODELOSMULTICRITERIO.pdf

12. Vidal, A. Modelo de valoración catastral de suelo y construcción mediante SIG para el Cantón San Cristóbal de Patate, Tungurahua, Ecuador [Universidad de Salzburg].

(2019). https://issuu.com/unigis_latina/docs/tes is_vidal1.

13. Vidal, R. El Método de los Valores Tipológicos (MVT). Ideas para un método de identificación y de prevaloración cualitativa de áreas a urbanizar. Revista de Geografía Norte Grande, (2008); 39(39-58).

https://scielo.conicyt.cl/scielo.php?scrip $\mathrm{t}=$ sci_arttext\&pid=S071834022008000100004

14. Gonzales, P., Villaronga, E., \& Turmo, J. La valoración inmobiliria: teoría y práctica. Wolters Kluwer. (2006). https://dialnet.unirioja.es/servlet/libro? codigo $=262182$

15. Martínez, M. Valoración del suelo. Método residual dinámico simplificado. Catastro, (2003). 47(5), 93-120. http://www.catastro.meh.es/documento s/publicaciones/ct/ct47/05CATASTR047.pdf

16. Aguado, $\mathrm{M}$. El régimen de valoración en el texto refundo de la Ley de Suelo. Revista Electrónica CEMCI, (2009); (2)1-32. https://revista.cemci.org/numero2/tribuna/articulo1.pdf

17. Martínez, T. El suelo: gestión y valoración [Universitat Politècnica de València].

(2014).

https://riunet.upv.es/bitstream/handle/ 10251/48868/TFG-ELSUELO-

GESTIONYVALORACION.pdf?sequence=1.

18. Miduvi. Norma técnica para la valoración de bienes inmuebles urbanos y rurales del Distrito Metropolitano de Quito. (2018).

http://www7.quito.gob.ec/mdmq_orden anzas/Circulares/2018/156-AVANCE CUMPLIMIENTO ORDM-196_VALOR DE LA TIERRA/ANEXO/NORMA_TÉCNICA_VAL ORACION_2019_miduvi.pdf

19. Recalde, A. Diseño y desarrollo de la aplicación "RH CIVIL" como herramienta para la valoración y control de avance de obras de bienes inmuebles urbanos mediante una base de datos, con requerimientos tipo de superintendencia de bancos [Escuela Politécnica Nacional]. (2017). https://bibdigital.epn.edu.ec/bitstream/ 15000/17592/1/CD-8073.pdf.

20. Carpio, G. Precio del suelo y metodologías de avaluación. Exploración para la captura de plusvalías urbanas en el caso de la Región Metropolitana de Santiago de Chile [Universidad de Chile]. (2014). http://repositorio.uchile.cl/bitstream/ha ndle/2250/130467/precio-del-suelo-ymetodologias-de- avaluacion.pdf; jsessionid=2C80F27FD1B22CF9985540A 0C9645F2B? sequence $=1$ 